\title{
Originales
}

\section{Traqueotomías percutáneas y coniotomías quirúrgicas en el paciente crítico}

\author{
L. MARRUECOS-SANT, L. ZAPATA-FENOR, E. MANERO-CABALLERO, P. VERA-ARTÁZCOZ \\ Y GRUPO DE TRABAJO DEL PROTOCOLO INSTITUCIONAL DE TRAQUEOTOMÍAS DEL HOSPITAL \\ DE LA SANTA CREU I SANT PAU*
}

Servicio de Medicina Intensiva. Hospital de la Santa Creu i Sant Pau. Barcelona. España.

Objetivo. Revisar y comparar las complicaciones de las traqueotomías percutáneas (TP) y las coniotomías por disección (CT) como métodos de canulación subglótica de la vía aérea.

Diseño. Estudio prospectivo y observacional, desde octubre de 2004 a octubre de 2006 y seguimiento de la evolución hasta mayo de 2007.

Ámbito. Servicio de medicina intensiva (SMI) de un hospital universitario.

Pacientes. 82 pacientes a los que se realizó canulación subglótica mediante TP (42 casos) o CT (39 casos).

Variables de interés. Motivo para la canulación subglótica, datos demograficos, gravedad, días de hospitalización en el SMI, días de intubación orotraqueal (IOT), problemas inmediatos y tardíos, evolución.

Resultados. Fueron canulados por ventilación prolongada $62(76 \%)$ pacientes y por depresión neurológica, el resto. No hubo diferencias entre TP y CT en el sexo, la gravedad de la enfermedad medida por APACHE II, los días de estancia en SMI y los días de IOT previos a la realización de la canulación subglótica. Los pacientes del gru-

*Grupo de Trabajo del Protocolo Institucional de Traqueotomías del Hospital de la Santa Creu i Sant Pau: Unidad de Broncología (A. TorregoFernández, M.C. Puzo-Ardanuy, C. Burgués-Mauri); Servicio de Medicina Intensiva (L. Marruecos-Sant); Servico de Neumología (P. Antón-Albisu, M.R. Güell-Rous); Servicio de Cirugía Torácica (C. León-González, G. Estrada-Saló, G. Gómez-Sebastian); Servicio de Otorrinolaringología (X. León-Vintró).

Correspondencia: Dr L. Marruecos-Sant

Servicio de Medicina Intensiva. Hospital de la Santa Creu i Sant Pau. Barcelona. España.

Sant Antoni Maria Claret, 167. 08025 Barcelona. España.

Correo electrónico: Lmarruecos@santpau.es

Manuscrito aceptado el 24-10-2008. po CT tuvieron más edad $(68 \pm 9$ frente a $54 \pm 15$ años; $p<0,001)$. Hubo 5 pacientes en el grupo TP con problemas leves $(3$ por angulación del fiador y 2 por punción traqueal lateral). Hubo 1 caso en el grupo CT con dificultad en la ventilación pulmonar durante el procedimiento. No hubo ningún fallecimiento relacionado con la TP o la CT. Fueron descanulados 34 pacientes; en 8 casos (6 TP y 2 CT) se objetivaron lesiones leves sin repercusión clínica. Se produjo un granuloma subglótico de forma tardía en un paciente con CT.

Conclusiones. En nuestra experiencia la CT es una alternativa a la TP cuando ésta no está indicada.

PALABRAS CLAVE: Traqueotomía. Coniotomía. Traqueotomía percutánea.

\section{PERCUTANEOUS TRACHEOTOMY AND CRIOTHYROIDOTOMY IN THE CRITICAL PATIENT}

Objective. To review and compare the complications of percutaneous tracheotomy (TP) and cricothyroidotomy (CT) used to perform tracheal intubation in patients requiring prolonged mechanical ventilation.

Design. A prospective, observational study performed from October 2004 to October 2006, and follow-up of course until May 2007.

Setting. Intensive care service from a university-affiliated teaching hospital.

Patients. A total of 82 patients in which CT or TP were necessary. Forty-three TP and 39 CT were performed.

Main measurements. Reason for TP or CT, demographic data, severity scores, ICU length of stay, orotracheal intubation (OTI) days, CT/TP early and late complications and in-hospital evolution were collected. 
Results. TP/CT were performed due to prolonged ventilation in $62(76 \%)$ patients and because of impaired neurological status in the remaining patients. There were no differences between TP/CT in gender, APACHE II, ICU length of stay, previous OTI days. Patients in the CT group were older $(68 \pm 9$ vs $54 \pm 15$ years, $p<0.001$ ). There were 5 mild adverse events ( 3 guide angulations and 2 lateral tracheal punctions) after TP, and 1 severe adverse event (pulmonary ventilation problem) after CT. There were no fatal event related with TP/CT. Thirty-four patients were decanulated. Mild local injuries were seen in 8 patients (6 TP vs 2 CT). Only 1 subglottic granuloma was seen late in CT group.

Conclusions. In our experience CT constitutes a safety and feasible alternative to TP when TP is counter-indicated.

KEY WORDS: Tracheotomy. Cricothyroidotomy. Percutaneous tracheotomy.

\section{INTRODUCCIÓN}

La traqueotomía, como canulación subglótica de la vía aérea, es una técnica indicada cuando el paciente requiere ventilación mecánica prolongada o el mantenimiento de la permeabilidad de la vía respiratoria. En comparación con la intubación traslaríngea, se preconiza que la traqueotomía se tolera mejor pues requiere menos analgesia-sedación, produce menos problemas en el aparato fonatorio, reduce el espacio muerto y resulta más estable y segura ${ }^{1-3}$, si bien no está demostrado que influya en la evolución clínica de los pacientes con ventilación mecánica ${ }^{2,4}$.

La traqueotomía percutánea (TP) es un método de utilización común en la actualidad en los servicios de medicina intensiva (SMI), como alternativa a la traqueotomía quirúrgica ${ }^{5-10}$. La TP permite que el personal propio del SMI pueda realizar la traqueotomía sin requerir el traslado del paciente y no depende de otro servicio, lo cual agiliza su realización y además reduce el tiempo y la agresión quirúrgica por sus propias características.

Otra alternativa a la traqueotomía quirúrgica y a la TP es la coniotomía por disección (CT). La CT es una técnica quirúrgica que consiste en la canulación subglótica de la vía aérea mediante la disección y apertura de la membrana cricotiroidea. Nos referimos a una técnica programada, no a la punción percutánea urgente o emergente de la membrana cricotiroidea ${ }^{11}$.

En el SMI de nuestro hospital, desde 2004 se realiza la permeabilidad subglótica de la vía aérea mediante la colocación de una cánula por TP como método prioritario o mediante una CT cuando la TP no es posible o no está indicada.

El presente trabajo se ha realizado con el objetivo principal de evaluar las diferencias entre las dos técnicas, TP y CT, en nuestra casuística reciente.

\section{PACIENTES Y MÉTODO}

Se estudiaron de forma prospectiva y observacional las canulaciones subglóticas de la vía aérea reali- zadas en el SMI de un hospital universitario en el período de octubre de 2004 a octubre de 2006. Se recogió información de las siguientes variables: motivo que indicó la canulación subglótica, datos demográficos, gravedad al ingreso medida mediante el APACHE II (Acute Physiology and Chronic Health Evaluation $)^{12}$, días de hospitalización en el SMI, días de intubación orotraqueal (IOT) previos a la TP/CT, problemas que se produjeron en la realización de la técnica, problemas tras la descanulación, evolución en el SMI y hospitalaria y mortalidad. Para conocer la evolución a largo plazo de los pacientes y de la traqueotomía, en mayo de 2007 se realizó una encuesta telefónica al domicilio de los pacientes. Se requirió la inclusión de un consentimiento informado para la realización de las TP y CT que recoge el estudio.

La indicación de una traqueotomía está observada en el protocolo institucional de nuestro hospital (Protocolo de Manejo del Paciente con Traqueotomía, enero de 2001) en las siguientes situaciones:

- Pacientes con enfermedades respiratorias crónicas y otras entidades patológicas que cursen con insuficiencia ventilatoria crónica y precisen ventilación mecánica invasiva.

- Pacientes con necesidad de protección de la vía aérea o su acceso para el drenaje de secreciones, que suelen ser pacientes con alguna neuropatía.

- Pacientes con intubación translaríngea prolongada (15-20 días).

- Pacientes con obstrucción de la vía aérea superior (básicamente pacientes controlados y tratados por el servicio de otorrinolaringología).

De las diversas técnicas existentes en la actualidad para realizar una TP, se optó por el método de Griggs, que utiliza como elemento dilatador de la tráquea una pinza-fórceps ${ }^{1}$.

Se realizó una CT si la TP estaba contraindicada por la existencia de algún factor de riesgo: intubación translarígea previa problemática (grado 3 o 4 de Mallampati, o grado 3 o 4 de Cormack y Lehane) ${ }^{13}$, características anatómicas propias que dificulten la identificación de los cartílagos cricoides y tiroides, obesidad, presencia de cuello corto con espacio cricoesternal $<3 \mathrm{~cm}$, bocio o vasos en la línea media.

Hasta la introducción de la TP en nuestro SMI, la CT ha sido la técnica habitual en la canulación subglótica de la vía aérea, practicada por el servicio de cirugía torácica en el mismo SMI, excepto si el paciente presentaba alguna lesión laríngea que indicase una traqueotomía quirúrgica. En el estudio actual la TP y la CT fueron realizadas por personal médico del SMI. La muestra actual engloba el período inicial de instauración de las dos técnicas, la TP y la CT, por personal del SMI.

En las $8 \mathrm{~h}$ previas al procedimiento se suspendió la dieta por vía entérica y la heparina que el paciente pudiese tener indicada. Durante todo el procedimiento de la TP/CT, practicado sin control broncoscópico concomitante, se monitorizaron la presión arterial sistémica, el ECG y la saturación arterial de $\mathrm{O}_{2}$. Un médico atendió exclusivamente la vía aérea translaríngea y la sedoanalgesia del paciente. Todas las TP/ 
CT se realizaron en pacientes hemodinámicamente estables y con necesidades de $\mathrm{FiO}_{2} \leq 50 \%$ y PEEP $\leq$ $5 \mathrm{cmH}_{2} \mathrm{O}$ previos al procedimiento. El procedimiento se realizó con $\mathrm{FiO}_{2}$ del $100 \%$ y sin PEEP.

Los problemas que pudieran surgir durante el procedimiento o posteriormente se dividieron en leves -desaturación arterial puntual (<60 s), angulación del fiador, punción lateral, sangrado menor $(<200$ ml) y celulitis- y graves -desaturación por dificultad en la canulación, sangrado mayor (> $200 \mathrm{ml})$, neumotórax, neumomediastino, enfisema subcutáneo, colocación inadecuada de la cánula, necesidad de una reconversión quirúrgica, reintubación translaríngea, lesión de la arteria innominada, fístula traqueoesofágica, lesión laríngea, laceración traqueal, estenosis traqueal, estenosis subglótica, parada cardiorrespiratoria y muerte-1,14-16.

En el estudio estadístico se aplicó la prueba de la t de Student y el test de la varianza (ANOVA) para comparar variables continuas. Para las variables cualitativas, el test de la $\chi^{2}$. Se consideró significación estadística si $\mathrm{p} \leq 0,05$.

\section{RESULTADOS}

Se presentan los datos correspondientes a los 82 pacientes con canulación subglótica de la vía aérea realizadas entre octubre de 2004 y octubre de 2006; se desglosan los datos generales y los correspondientes a las $43 \mathrm{TP}$ y las $39 \mathrm{CT}$, mientras se obvian las otras técnicas que se realizaron (1 traqueotomía quirúrgica y 4 coniotomías percutáneas).

En el mismo intervalo que duró el estudio fueron atendidos en el SMI 888 pacientes, con una media de edad de $62 \pm 16$ años y un APACHE II al ingreso en el SMI de $20 \pm 10$. Requirieron intubación translaríngea durante más de 48 h 533 (60\%) pacientes, por lo que los 82 pacientes con canulación subglótica de la vía aérea suponen el 15,3\% de los pacientes intubados por vía translaríngea y el 9,2\% del total de pacientes atendidos durante el periodo de estudio. La mortalidad en el SMI en este periodo fue del 32,4\% del total de pacientes ingresados, el 31,8\% de los 533 pacientes intubados y el $34,1 \%$ de los 82 pacientes estudiados (tabla 1).
En la tabla 2 se muestran las caractísticas de los 82 pacientes que requirieron canulación subglótica con TP o CT. La edad de los pacientes del grupo de CT fue significativamente mayor que la de los pacientes con TP, y en ambos grupos predominaron los varones.

El diámetro interno de las cánulas empleadas fue 8 mm en $72(82,7 \%)$ casos, $7 \mathrm{~mm}$ en 8 y $8,5 \mathrm{~mm}$ en 2 . Todas las cánulas de $7 \mathrm{~mm}$ se sustituyeron posteriormente por una cánula de $8 \mathrm{~mm}$.

En $6(7,3 \%)$ casos hubo un problema durante la realización de la TP/CT, que en 5 se valoró como leve; 3 fueron por angulación del fiador y 2, por punción traqueal lateral, los 5 al realizar una TP.

El único problema calificado como grave, o potencialmente grave, se produjo en una CT que se realizó sin problemas. Consistió en la dificultad para ventilar al paciente al colocar la cánula traqueal. Por control broncoscópico se objetivó que, por angulación de la cánula y la disposición de la tráquea del paciente, se producía una obstrucción del orificio de la cánula al contactar con la pared anterior de la tráquea. El paciente en todo momento estuvo ventilado y oxigenado sin problemas mediante el tubo translaríngeo. El problema se solucionó colocando una cánula de menor angulación.

TABLA 1. Datos generales de los pacientes
atendidos en el SMI en el periodo octubre
de 2004 a octubre de 2006 y de los pacientes que
requirieron canulación subglótica de la vía aérea
en el mismo periodo
\begin{tabular}{|l|c|c|}
\hline \multicolumn{1}{|c|}{$\begin{array}{c}\text { Pacientes con } \\
\text { Pacientes ingresados } \\
\text { en el SMI }(\mathrm{n}=888)\end{array}$} & $\begin{array}{c}\text { culación subglótica } \\
(\mathrm{n}=82)\end{array}$ \\
\hline $\begin{array}{l}\text { Intubación orotraqueal } \\
>48 \mathrm{~h}, \mathrm{n}(\%)\end{array}$ & $533(60)$ & $82(100)$ \\
$\begin{array}{c}\text { Edad (años), } \\
\text { media } \pm \text { DE }\end{array}$ & $62 \pm 16$ & $61 \pm 14$ \\
$\begin{array}{c}\text { APACHE II, } \\
\text { media } \pm \text { DE } \\
\text { Mortalidad en el SMI }\end{array}$ & $19 \pm 10$ & $20 \pm 10$ \\
\hline
\end{tabular}

DE: desviación estándar; SMI: servicio de medicina intensiva.

TABLA 2. Características de los 82 pacientes con canulación subglótica incluidos en el estudio

\begin{tabular}{|l|c|c|c|}
\hline & $\begin{array}{c}\text { Traqueostomía percutánea } \\
(\mathrm{n}=43)\end{array}$ & Coniotomía $(\mathrm{n}=39)$ & $\mathrm{p}$ \\
\hline Edad (años) & $54 \pm 15$ & $68 \pm 9$ & $<0,001$ \\
Varones/mujeres, n (\%) & $32(75) / 11(25)$ & $31(79) / 8(21)$ & NS \\
APACHE II & $19 \pm 8$ & $22 \pm 11$ & NS \\
Motivo para canulación subglótica & $31(72)$ & $31(79)$ & $8(21)$ \\
Ventilación prolongada, n (\%) & $12(28)$ & - & $19 \pm 8$ \\
Coma, n (\%) & - & $37 \pm 16$ & NS \\
Otras & $18 \pm 10$ & NS \\
Días de intubación orotraqueal & $39 \pm 36$ & \\
Días de estancia en el SMI & & \\
\hline
\end{tabular}

DE: desviación estándar; SMI: servicio de medicina intensiva.

Los datos expresan media \pm DE excepto donde se indica 
En el SMI fallecieron 28 de los 82 pacientes por la evolución de su enfermedad (tabla 1). En sala de hospitalización convencional fallecieron posteriormente 6 pacientes, y otros 9 en el centro hospitalario al que fueron trasladados, ninguno por un problema relacionado con la TP o la CT. Se pudo descanular a 6 pacientes en el SMI y después a otros 14 en sala convencional durante el mismo ingreso hospitalario. Por los datos aportados mediante la encuesta realizada en mayo de 2007, se constató que tras el alta de nuestro hospital fueron descanulados otros 14 pacientes. En definitiva, $43(52,4 \%)$ pacientes fallecieron y de los 39 restantes, fueron descanulados $34(41 \%)$. De los 5 $(6 \%)$ restantes no se obtuvo información de su evolución.

En los 20 pacientes que fueron descanulados durante el ingreso hospitalario, el tiempo de canulación fue de $34 \pm 32$ días. No hubo problemas tras la descanulación en estos 20 pacientes.

En 10 pacientes la descanulación se realizó con control broncoscópico y se objetivaron $8(9,7 \%)$ lesiones leves: 3 lesiones en el estoma cutáneo ( 2 TP, 1 CT), 2 en la glotis (1 TP, 1 CT) y 3 lesiones superficiales en la tráquea (3 TP). Ninguna de estas lesiones tuvo repercusión clínica.

Los días de IOT previos a la descanulación no mostraron ninguna relación con las lesiones objetivadas (estoma: $11 \pm 5$; glóticas: $15 \pm 14$; traqueales: $6 \pm$ 2; ninguna: $20 \pm 7$ ).

En los 34 pacientes descanulados se detectó, mediante laringoscopia realizada en septiembre de 2006 , un granuloma subglótico de la pared posterior en un paciente que no presentó ninguna lesión en el control broncoscópico inmediato a su descanulación en febrero de 2006, tras ser portador de una cánula de coniotomía durante 20 días, y había requerido tres intubaciones previas por ventilación prolongada, con un tiempo de IOT de 21 días. El granuloma no requirió resección. Los demás pacientes descanulados no presentaron problemas.

\section{DISCUSIÓN}

La muestra de 82 pacientes con canulación subglótica de la vía aérea fue similar en edad y gravedad a la total de los pacientes atendidos en el SMI durante el mismo periodo, es decir, no constituye un subgrupo de pacientes distinto del total de la población atendida en el SMI en cuanto a las variables descritas. Ni el diseño del estudio ni el tamaño de la muestra pretenden analizar la influencia de la canulación en la evolución de los pacientes, aspecto que actualmente no está establecido en la literatura ${ }^{2,4}$.

En nuestra serie, un $15,3 \%$ de los pacientes intubados más de 48 h presentaron indicación para realizar TP o CT, porcentaje que varía en las distintas series en función de las indicaciones de canulación subglótica de la vía aérea que tuvieran establecidos y el tipo de enfermos atendidos. En la serie de Madero-Pérez et $\mathrm{al}^{10}$, de 115 casos con TP, ésta se indicó en el $9 \%$ de los pacientes con ventilación mecánica y el motivo de la canulación fue en un $56 \%$ la ventilación prolon- gada y en un $29 \%$ las alteraciones en el nivel de conciencia.

No hay evidencia sobre la influencia de los distintos procedimientos de canulación subglótica de la vía aérea frente a la intubación translaríngea ${ }^{3,4,17}$.

De las diversas técnicas de TP que hoy existen, ninguna da mayor seguridad o mejores resultados, por lo que se aconseja elegir y dominar una de las diversas variedades ${ }^{1,8}$; en nuestro servicio se ha optado por el método de Griggs. En un estudio de una encuesta nacional realizado en España en 2002, fue el método más frecuentemente utilizado ${ }^{5}$.

La CT es una técnica que fue desaconsejada en 1921 y rehabilitada en sus indicaciones en 1976 para el caso de que haya alguna dificultad anatómica para la realización de una traqueotomía (cuello corto, cifosis cervical, tráquea profunda, obesidad mórbida) $)^{18-20}$. En el metaanálisis de Burkey et $\mathrm{al}^{19}$ de 1991 y en la serie de Bruno et $\mathrm{al}^{20}$ de 2003 , se publica la experiencia con esta técnica, pero no existen estudios comparativos aleatorizados. Se considera que sus ventajas son la facilidad de acceso, la rapidez en su realización y que es practicable en la cama del enfermo. Se estima que produce menos complicaciones generales por estar más distante de otras estructuras del cuello (vasos, tiroides, mediastino) y el esternón ${ }^{14,18-20}$. Algunos autores han preconizado la realización percutánea de la coniotomía ${ }^{21,22}$ como alternativa a la TP, aunque son series de 44 y 100 pacientes respectivamente.

En nuestra serie los dos subgrupos estudiados, TP y CT, fueron similares en cuanto a la distribución por sexos, gravedad (APACHE II), motivo de la indicación de TP/CT y en los días de atención en el SMI. En cuanto a la edad, se apreció una diferencia estadísticamente significativa entre las TP y las CT; los pacientes a los que se realizó una CT eran mayores, por lo que se puede interpretar que los factores de riesgo que establecieron la indicación de una CT son más frecuentes a mayor edad de los pacientes.

Los días de IOT previos fueron similares en los dos grupos. No existe un número de días determinado para indicar la realización de una TP/CT. Se define como indicación precoz cuando es en los primeros 7 días $^{3,4}$. Algunos trabajos han propuesto la precocidad en la canulación como una medida que posibilitaría la reducción del tiempo de ventilación mecánica y de estancia en el SMI ${ }^{23}$. Diversas publicaciones establecen entre 12 y 21 días el tiempo de IOT orientativo $^{1-3,10,17,23,24}$.

De los 6 problemas detectados durante la realización de cada una de las técnicas de canulación, 5 fueron valorados como leves; 3 fueron por angulación del fiador, sin ninguna consecuencia, y 2 por punción lateral, también sin consecuencias, y una de las punciones laterales se sustituyó por una CT. Las dos punciones laterales se detectaron por observación directa durante la realización del procedimiento. Una se corrigió realizando una nueva punción y la otra fue sustituida por una CT, ya que el espacio cricoesternal estaba justo en el límite de $3 \mathrm{~cm}$, y además el cuello era muy adiposo. Las 5 complicaciones leves se produjeron durante el periodo inicial de instauración de 
la técnica de TP en nuestro SMI. Se considera que con un control broncoscópico durante la realización del procedimiento se puede reducir el número de problemas $^{1,5}$, y es aconsejable añadir control broncoscópico en los periodos de aprendizaje y también al indicar una TP si hay factores de riesgo, lo cual podría disminuir la indicación de CT en nuestra casuística.

Como complicaciones más frecuentes durante la realización de una TP, se han descrito hemorragias locales leves, con una frecuencia de un $9-13 \%$. los periodos de instauración de las TP las complicaciones mayores (hemorragias graves, fístulas traqueoesofágicas, neumotórax, neumomediastino) pueden ser menos frecuentes si se realizan con control broncoscópico, pues se han descrito tasas de hasta un $4 \%{ }^{1,5,6,10}$. Las hemorragias tardías pueden observarse en un $3 \%$, de las que alrededor de un $10 \%$ es por lesiones del tronco innominado ${ }^{16}$.

Los problemas observados tras la descanulación, valorados con control broncoscópico en 10 pacientes y de una forma tardía por encuesta telefónica en los 34 pacientes descanulados, expresan que el porcentaje de complicaciones es escaso. En nuestra casuística el control tardío no detectó problemas con repercusión clínica que pudieran haber requerido alguna exploración complementaria, generalmente endoscópica, si bien las lesiones sin expresividad clínica pueden haberse producido sin que se las detectara con el método de encuesta telefónica empleado. Las complicaciones no guardaron ninguna relación con el tiempo previo de IOT, si bien en nuestra muestra éste fue reducido.

En relación con la CT, la principal complicación posible es la estenosis subglótica. Como factores de riesgo de que se produzca una estenosis subglótica, se han valorado la existencia de afección laríngea previa a la canulación (infección, tumor) y la intubación translaríngea prolongada. En la revisión de Burkey et $\mathrm{a}^{19}$, en las CT las estenosis subglóticas alcanzaban el $4 \%$ cuando había factores de riesgo y el $1 \%$ sin factores de riesgo.

En la literatura se considera que la causa más frecuente de estenosis subglótica es un tiempo de IOT > 8 días ${ }^{11,14,25,26}$. El tiempo de IOT está relacionado con lesiones secundarias como la estenosis traqueal y la estenosis subglótica, en pacientes no canulados y canulados, ya sea por traqueotomía quirúrgica, TP o por $\mathrm{CT}^{14,15,19,25}$. En el trabajo de Koitschev et $\mathrm{al}^{15}$, pese a no tener repercusión clínica, se objetivaron estenosis traqueales en un 58\% de las TP y un $24 \%$ de las traqueotomías quirúrgicas ${ }^{15}$. Tampoco no se observaron diferencias en los días de IOT en relación con las lesiones objetivadas tras la descanulación.

En el caso descrito en nuestra serie de estenosis subglótica tardía por granuloma, el tiempo de IOT fue algo mayor que la media observada en el resto de los pacientes (21 frente a 18,8 días), y el paciente había sido intubado por vía translaríngea tres veces sucesivas durante el mismo ingreso, lo que podría ser un importante factor inductor del granuloma. En la serie de Bruno et $\mathrm{al}^{20}$ no se observaron diferencias entre 86 traqueotomías y 32 coniotomías en las complicaciones que presentaron, con 1 caso de estenosis subglótica en cada grupo. En 1991, la revisión de Burkey et $\mathrm{al}^{19}$ de 17 trabajos publicados desde 1976 a 1989 subraya la importancia de la afección laríngea previa y el tiempo de IOT como los factores más importantes relacionados con las lesiones subglóticas que puedan producirse. En la casuística de Barrachina et $\mathrm{al}^{22}$, con 44 CT percutáneas, el tiempo de IOT fue de 15 (2-44) días, con una media de tiempo de canulación de 26 días, y no se objetivó ninguna estenosis, aunque sí 1 caso de infección del estoma; en 13 casos se realizó control broncoscópico posterior a la descanulación. También están descritas disfunciones laríngeas secundarias a las $\mathrm{CT}^{19,27}$.

El diámetro interno de las cánulas utilizadas fue predominantemente de $8 \mathrm{~mm}$, de forma indistinta si eran en una TP o una CT, con un diámetro externo de $11 \mathrm{~mm}$. En las CT se realiza la apertura de la membrana cricotiroidea, que tiene un tamaño que oscila alrededor de $30 \mathrm{~mm}$ de amplitud por unos $10 \mathrm{~mm}$ de altura, y se aconseja utilizar cánulas con un diámetro externo alrededor de $10 \mathrm{~mm}^{11,14,19}$, con el fin de reducir el riesgo de lesiones laríngeas consecuentes a este procedimiento.

La mortalidad fue similar a la de los pacientes intubados por vía translaríngea. Todos los pacientes fallecieron por causas no relacionadas con TP o CT, de forma precoz o tardía. La posibilidad de muerte durante las técnicas de TP o CT está descrita en relación con las complicaciones graves ${ }^{1,11,14-16}$. En la serie de Frutos-Vivar et a ${ }^{17}$ con 546 pacientes con traqueotomía, la mortalidad en el SMI fue menor que entre los pacientes no traqueotomizados, pero con igual mortalidad hospitalaria, a diferencia de nuestra casuística, en la que la mortalidad de los pacientes con traqueostomía no fue diferente de la del resto de los pacientes intubados.

En conclusión, en la serie estudiada, si bien el tamaño de la muestra es pequeño y no se la ha aleatorizado, no hay diferencia entre el periodo inicial y el tardío en las complicaciones producidas con las dos técnicas empleadas, y son parecidas a las reflejadas en la literatura revisada. La CT puede constituir una alternativa a la CT cuando no es factible la indicación de una TP.

\section{BIBLIOGRAFÍA}

1. Kost KM. Endoscopic percutaneus dilatational tracheotomy: a prospective evaluation of 500 consecutive cases. Laryngoscope. 2005;115:1-30.

2. Combes A, Luyt CE, Nieszkowska A, Trouillet JL, Gibert C, Chastre J. Is tracheostomy associated with better outcomes for patients requiring long-term mechanical ventilation? Crit Care Med. 2007;35:802-7.

3. Clum SR, Rumbak MJ. Mortality and tracheotomy. Crit Care Med. 2007;35:963-4.

4. Clec'h C, Alberti C, Vincent F, Garrouste-Orgeas M, Lassence A, Toledano D, et al. Tracheostomy does not improve the outcome of patients requiring prolonged mechanical ventilation:A propensity analysis. Crit Care Med. 2007;35:132-8. 
5. Añón JM, Escuela MP, Gómez V, García A, Montejo JC, López J. Use of percutaneus tracheostomy in intensive care units in Spain. Results of a national survey. Intensive Care Med. 2004;30:1212-5.

6. Freeman BD, Isabella K, Lin N, Buchman TG. A meta-analysis of prospective trials comparing percutaneous and surgical tracheostomy in critically ill patients. Chest. 2000;118:1412-8.

7. Silvester W, Goldsmith D, Uchiono S, Bellomo R, Knight $\mathrm{S}$, Seevanayagam S, et al. Percutaneous versus surgical tracheostomy: a randomized controlled study with long-term followup. Crit Care Med. 2006;34:2145-52.

8. Castella-Picas FX. La traqueotomía diez años después. Med Intensiva. 2000;24:348-52.

9. Doménech I, Mateu T, Cisa E, Juan A, Gil E, Palau M, et al. Traqueotomía percutánea por dilatación: nuestra experiencia. Acta Otorrinolaringol Esp. 2004;55:334-7.

10. Madero Pérez J, Vidal Tegedor B, Abizanda Campos R, Cubedo Bort M, Alvaro Sánchez R, Micó Gómez M. Traqueostomía percutánea en pacientes ventilados. Med Intensiva. 2007;31:120-5.

11. Esses BA, Jafek BW. Cricothyroidotomy: a decade of experience in Denver. Ann Otol Rhinol Laryngol. 1987;96:519-24.

12. Knaus WA, Draper EA, Wagner DP, Zimmerman JE. APACHE II: A severity of disease classification. Crit Care Med. 1985;13:818-29.

13. Shiga T, Wajima Z, Inoue T, Sakamoto A. Predicting difficult intubation in apparently normal patients: a meta-analysis of bedside screening test performance. Anesthesiology. 2005;103:429-37.

14. Boon JM, Abrahams $\mathrm{PH}$, Meiring $\mathrm{JH}$, Welch $\mathrm{T}$. Cricothyroidotomy: a clinical anatomy rewiev. Clin Anat. 2004; 17:478-86.

15. Koitschev A, Simon C, Blumenstock G, Mach H, Graumuller S. Suprastomaltracheal stenosis after dilational and surgical tracheostomy in critically ill patients. Anaesthesia. 2006;61:832-7.

16. Espeel B, Buron F, Lismonde M, Lambot D, Fredericky Y. Massive bleeding due to a brachiocephalic trunk erosion during a percutaneous tracheotomy. Intensive Care Med. 2006;32:943-4
17. Frutos-Vivar F, Esteban A, Apezteguia C, Anzueto A, Nightingale P, Gonzalez M, et al. Outcome of mechanically ventilated patients who require a tracheostomy. Crit Care Med. 2005;33:290-8.

18. Wenig BL, Applebaum EL. Indications for and techniques of tracheotomy. Clin Chest Med. 1991;12:545-53.

19. Burkey B. Esclamado R, Morganroth M. The role of cricothyroidotomy in airway management. Clin Chest Med. 1991;12:561-71.

20. Bruno F, Clavel M, Desachy A, Puyraud S, Roustan J, Vignon P. Complications of tracheostomy performed in the ICU. Chest. 2003;123:151-8.

21. Toye FJ, Weinstein JD. Clinical experience with percutaneous tracheostomy and cricothyroidotomy in 100 patients. J Trauma. 1986;26:1034-40.

22. Barrachina F, Guardiola JJ, Añó T, Ochagavia A, Mariné J. Percutaneous dilatational cricothyroidotomy: outcome with 44 consecutive patients. Intensive Care Med. 1996;22:937-40.

23. Griffiths J, Barber VS, Morgan L, Young JD. Systematic review and metaanalysis of studies of the timing of tracheostomy in adult patients undergoing artificial ventilation. BMJ. 2005;330:1243-7

24. Freeman BD, Borecki IB, Coopersmith CM, Buchman TG. Relationship between tracheotomy timing and duration of mechanical ventilation in critically patients. Crit Care Med. 2005;33:2513-20

25. Pino Rivero V, Marcos García M, Trinidad Ruíz G, Pardo Romero G, González Palomino A, Keituqwa Yánez T, et al. Estenosis laringo-traqueal en adultos. Estudio retrospectivo de 21 casos y revisión de la literatura. Acta Otorrinolaringol Esp. 2004;55:376-80.

26. Heffner JE. Airway management in the critically ill patient. Crit Care Clin. 1990;6:533-51

27. Lim JW, Lerner PK, Rothstein SG. Epiglotic position after cricothyroidotomty: a comparison with tracheotomy. Ann Otol Rhinol Laryngol. 1997;106:560-2. 